eclipse of 1926, where he was a member of the British Expedition to Benkoelen.

In Kedah or Siam there will probably also be an American expedition from Harvard, Prof. Stetson and Mr. Weld Arnold, with a programme of photometry-absolute and relative-and direct photography of the corona; and near Khoke Bhode, in Siam, there may also be a German expedition from Kiel and Göttingen. Their programme will include photometry and spectrophotometry of the chromosphere and corona and a search for faint coronal lines with a spectrograph of high light-gathering power.

On Poulo Condore, an island off the coast of Cambodia, there will be a French expedition from the Bureau des Longitudes. Coronal photometry and the Einstein experiment will form the main programme. At Iloilo, in the Philippines, a German expedition from Hamburg will be established with a programme including objective prism flash spectra and direct photography of the corona with a series of exposures of different lengths. It is possible that Anderson's apparatus may also be taken to the Philippines by a joint American and Norwegian expedition, mainly from the Naval Observatory, Washington.

The weather prospects along the whole belt are reasonably good, and with so many parties so well spaced along the belt of totality, it may be hoped that important results will be obtained in the whole field of eclipse problems of present-day interest.

\title{
The Structure of Atomic Nuclei.
}

$\mathrm{I}^{\mathrm{N}}$ opening the discussion on atomic nuclei. held at the Royal Society meeting on Feb. 7, the president, Sir Ernest Rutherford, directed attention to a former meeting, held at the Royal Society in March 1914, when the existing evidence on the nuclear structure of the atom was set out. The speakers at that meeting included the president as opener of the discussion, Moseley, Soddy, Nicholson, Hicks, H. S. Allen, and Sylvanus Thompson. It is of interest to note that at this meeting Moseley gave his final conclusion on the classification of the elements by their ordinal numbers, and that Soddy, after giving the evidence for the existence of radioactive isotopes, suggested that many of the ordinary elements might also consist of isotopes, a result so completely confirmed in later years.

The experiments described at that meeting in 1914 tended to show that the nucleus was to be regarded as a point, but in the intervening years evidence from a variety of sources has been accumulated which throws light on the structure of this minute central body. Sir Ernest directed attention to the three main lines of attack: the measurements of the masses of atoms, the evidence from collisions of $a$-particles with nuclei, and the evidence provided by the natural disintegration of the radioactive elements. He emphasised that, while many nuclear phenomena have been observed and investigated in the last decade, only one way of influencing the nucleus directly has been discovered. Although many attempts have been made to disintegrate the elements artificially, the only agents which have as yet accomplished this are the $\alpha$-particles emitted by radioactive bodies. The $a$-particles are helium nuclei with energies as high as seven million electron-volts, and, when their direction of impact on an atom is central, they can penetrate the atom and collide with the nucleus, thereby disintegrating it.

One of the most fruitful lines of investigation has proved to be the observation of the deflection $\alpha$ particles suffer when they pass near the nucleus but yet do not disintegrate it. This scattering, as it is termed, is due to the electrical forces between the a-particle with two elementary charges, and the nucleus with $Z$ elementary charges, where $Z$ is the atomic number. The $\alpha$-particles which penetrate closest to the nucleus are most deflected, so that conversely by observing the relative number of particles deffected at a certain angle, information can be obtained about the electrical forces between the particles for a definite distance of approach. It can be shown that the variation of the scattering at a definite angle as the velocity of the particle is changed gives direct information on the rate of variation of the electrical forces with distance.

An extended series of experiments on these lines has been made by Rutherford and Chadwick, and the results have shown that for the elements from copper (atomic number 29) to uranium (atomic number 92), the law of force is that of the inverse square. The closest distance of approach of the particles to the nucleus in these experiments was $10^{-12} \mathrm{~cm}$. in the case of copper, and about $4 \times 10^{-12}$ $\mathrm{cm}$. with uranium. The fact that no deviations from the inverse square were found indicates that for these distances of approach the two charged bodies are acting as points, and no information can be deduced about the dimensions of the nucleus except that it must be smaller than these distances. With lighter elements quite different results are obtained. Owing to the smaller nuclear charges, the $a$-particle can approach much nearer to the nucleus and marked deviations from the scattering expected on the inverse square law are observed. The most natural explanation of the results is found to be that, at very close distances, attractive forces come into play varying rapidly with the distance. The experiments are not as yet sufficiently definite to determine the rate of variation in detail. Debye and Hardmeier have attempted to put the existence of these forces on a physical basis by suggesting they are due to distortion or the mutual polarisation of the colliding particles. It appears that this hypothesis can give a general explanation of the scattering by light elements.

An extremely important application of these scattering experiments is obtained by considering the results with uranium. On ordinary views, part at least of the energy of the $\alpha$-particle ejected from the uranium nucleus must be due to the repul- 
sion of the inverse square law forces. If its entire energy is attributed to this cause, a minimum estimate will be obtained for the distance at which the inverse square force of repulsion begins to be appreciably diminished by the attractive forces. The calculation yields a value of $6 \times 10^{-12} \mathrm{~cm}$.; this is in complete disagreement with the scattering result already quoted, which showed that the inverse square field extended down to a distance of less than $4 \times 10^{-12} \mathrm{~cm}$. This impasse is avoided if we may make use of the wave-mechanics.

Dr. J. Chadwick described a similar phenomenon which is found to occur with aluminium, which is almost at the other end of the table of elements. Aluminium is one of the elements which can be disintegrated by the impact of $\alpha$-particles, and, as would be expected, the probability of disintegration decreases as the speed of the a-particle is decreased. Measurable disintegration, however, is still observed with $a$-particles of such low speed that the scattering observed in other experiments is still due to inverse square law forces. In one experiment, $\alpha$-particles of this speed appear to be able to hit the nucleus so as to disintegrate it, but yet in the other the same speed $\alpha$-particles are deflected as if the nucleus acted as a point charge.

Both these results can be explained at least qualitatively, according to the wave-mechanics, in a manner suggested independently by Gurney and Condon, and by Gamow. It is supposed that the repulsive inverse square law field surrounding the nucleus extends down to very small distances, of the order of $0.7 \times 10^{-12} \mathrm{~cm}$. for uranium, and rises to a peak value of the order of thirty million volts. The scattering results are therefore directly understandable. The reason why a slow particle can escape from the uranium nucleus, or in the other case penetrate into the aluminium nucleus, is to be sought for in the peculiar properties ascribed to particles by the wave-mechanics. On the classical theories, the only way a particle can pass from one region into a second separated from the first by a potential barrier is by surmounting the barrier. On the wave-theory, however, there is a finite probability of the particle passing through the potential barrier although its energy may be far less than the peak value.

Several points of exceptional interest have been discovered in connexion with the artificial disintegration of the elements. With the exception of lithium, beryllium, carbon, and oxygen, all the elements up to potassium can be disintegrated by the $\alpha$-particles of radium- $C$ (energy seven million electron volts). Particular attention had been directed to the energy relations occurring in the collision. For example, it is found that protons are knocked off the aluminium nucleus with energies as high as 1.4 times that of the incident $\alpha$-particle. The experiments of Blackett have shown that in the process of artificial disintegration of nitrogen, while a proton is knocked off, the $\alpha$-particle appears to be captured. If this is also the case in the disintegration of aluminium, it is possible to deduce from the experiments that what may be termed the heat of the reaction is not constant, but varies over a comparatively wide range. The obvious suggestion is that the masses of all aluminium nuclei are not identical.

The elements of odd atomic number give protons of greater range than those of even atomic number, and this distinction between the two classes was emphasised by Dr. F. W. Aston in connexion with his experiments on the isotopic constitution and masses of the elements. While the even atomic numbers often have many isotopes (tin has eleven), the elements of odd atomic number appear never to have more than two.

Valuable evidence on the stability of the elements is provided by the accurate measurements of their masses by the mass-spectrograph. The mass of a nucleus is in general found to be less than the sum of the masses of the protons, $\alpha$-particles, and electrons of which it is supposed to be constituted. This disappearance of mass represents an emission of energy in its formation, or conversely, that energy must be supplied to disintegrate it. The measurements so far carried out support both the results on the artificial disintegration and the occurrence of natural disintegration for elements of high atomic number. For example, with the radioactive elements it appears likely, as was pointed out by Sir Ernest Rutherford, that the $a$-particle has more mass inside the nucleus than when it is free.

Mr. R. H. Fowler gave an account of the developments of Gamow's theory, which has already been referred to. It has been seen how the experimental evidence leads to the conception of attractive forces at close distances giving rise to a potential barrier surrounding the nucleus as shown in Fig. 1. The problem is how constituents of

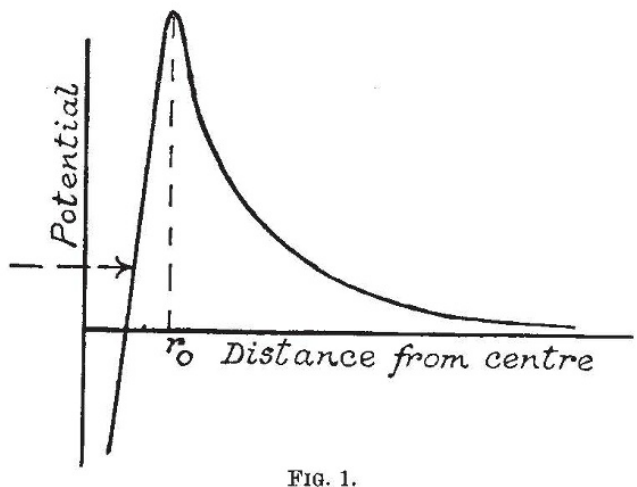

FIG. 1.

the nucleus such as a-particles which are inside this nucleus can escape, when their energy as represented by the arrow is insufficient to take them over the peak. An $\alpha$-particle inside such a nucleus is not to be considered as a material particle executing some kind of orbital motion, but as a wave motion filling the whole of the space. The wavelength is determined by the momentum of the $a$ particle, and as a rough illustration, the stable states are those in which some type of stationary wave system exists. If the potential barrier were infinitely thick or high, it would be possible to have a true stationary wave motion and the 
system would be permanently stable. With a finite barrier, the wave motion on the new mechanics is found to be a damped vibration coupled with an issuing wave which penetrates the barrier. The exact optical analogy is a wave incident at an angle greater than the critical angle on a surface only a few wave-lengths thick. In this case it has been shown experimentally that a small amount of the wave penetrates the surface. The issuing wave in the nuclear problem must be interpreted as showing that the $a$-particle has a possibly small, but yet finite chance of escape from the system with the same energy that it has inside the nucleus. This is in itself the explanation of radioactive disintegration, but the calculation can be pushed much further. It will be seen from Fig. 1 that the greater the energy of the $\alpha$-particle the smaller the thickness and height of the potential barrier it has to penetrate, and the greater should be the chance of escape, that is, disintegration. This is the well-known Geiger-Nuttall law connecting the energy of the ejected $\alpha$-particle with the transformation constant. Considering the provisional state of the theory, an excellent quantitative account can be given of the observed connexion between the energies of the $\alpha$-particles and the transformation constants. The distance $r_{0}$, about $0.7 \times 10^{-12} \mathrm{~cm}$. for heavy nuclei, may be looked on as the size of the nucleus, and it is found necessary to allow this to decrease as we go down the series. This is, of course, reasonable.

It has as yet not been possible to include the nuclear electrons in this theory, or to attempt an account of the $\beta$ - and $\gamma$-ray phenomena. The evidence on this subject was described by Dr. C. D. Ellis. The $\gamma$-rays are high frequency electromagnetic radiations emitted immediately after the departure of the disintegration particle and can be considered as the result of the adjustment of the nucleus to the new conditions. They constitute the characteristic spectrum of the nucleus and will no doubt provide valuable information on its structure, but at present there is difficulty in deciding how they are emitted. It appears unlikely that they can be emitted by electrons, but there is still the choice between the positive particles and the nucleus as a whole by some process such as rotation. There is clear evidence that the $\gamma$ rays can be associated with a level system, and while it may be difficult to fix the system from pure energy considerations, measurements of the intensities should enable a decision to be reached.
A phenomenon indicating a coupling between the nucleus and the outer electronic structure has been observed by G. H. Aston and Ellis. The energy of the excited nucleus is not always emitted in the form of radiation; it is sometimes converted inside the same atom and leads to the ejection of an electron from the atom. If the probability of this happening were to vary smoothly with the frequency, the process would be similar to the actual emission of the energy from the nucleus and reabsorption by the electrons. The two systems cannot be considered distinct in this way, since the probability of conversion is found to oscillate rapidly in ascending the scale of frequencies.

Prof. O. W. Richardson pointed out that spectroscopic evidence has an important bearing on the question whether the nucleus is in rotation. $\mathrm{He}$ described an a priori argument which makes it probable that the nuclei of the elements must in many instances be rotating. In the case of hydrogen, the result of this argument is almost a certainty. A universe is imagined which at a certain instant consists of one electron and one proton, and these unite to form an unexcited hydrogen atom with the emission of radiation. The spectroscopic evidence is overwhelming that in the ground state of an atom each extra-nuclear electron has half a quantum of angular rotation. If the postulated universe is to obey the principle of the conservation of angular momentum, the nucleus of the hydrogen atom must have acquired half a quantum of angular momentum in the opposite sense. An improbable but interesting alternative is that the emitted radiation preserves the conservation of momentum by having a sufficiently high degree of elliptical polarisation.

There is good spectroscopic evidence that the nuclei of a number of elements are rotating and have a quantised angular momentum. This is shown from the magnetic field resulting from this rotation, which causes the hyper-fine structure of the spectral lines of many atoms. A sufficient analysis has already been made for bismuth and crsium to indicate the exact number of quanta on the nucleus. The spectroscopic evidence of the alternating intensities in the band spectrum of hydrogen, and the specific heat of hydrogen gas, when interpreted according to the wave-mechanics, also definitely require the proton to have half a quantum of angular momentum.

\section{Obituary.}

Dr. H. J. H. Fenton, F.R.S.

$\mathrm{T}$ HE death of Dr. H. J. H. Fenton, formerly lecturer and demonstrator of chemistry in the University of Cambridge, will be regretted by many generations of Cambridge men, for he taught in the University for more than forty years, and of the numbers that have attended his lectures there can be few who did not receive a lasting impression from his teaching.
Henry John Horstmann Fenton was born at Ealing in 1854. After having been educated at Magdalen College School, Oxford, he went to King's College, London, where he studied chemistry under Bloxam, and at the end of his course acted as demonstrator. About this time the Clothworkers' Company instituted an exhibition in physical science tenable for three years by a noncollegiate student at Cambridge. The first award 